\title{
Prevalence and Predictors of Social Support Utilization among Cancer Patients Undergoing Treatment John Hunninghake, M.D. ${ }^{1}$, Fanglong Dong, Ph.D. ${ }^{2}$, Robert B. Hines, Ph.D. ${ }^{2}$, Elizabeth Ablah, Ph.D. ${ }^{2}$, Sarah Taylor, M.D. ${ }^{3}$ \\ ${ }^{1}$ San Antonio Military Medical Center, San Antonio, TX \\ ${ }^{2}$ University of Kansas School of Medicine-Wichita \\ Department of Preventive Medicine and Public Health \\ ${ }^{3}$ University of Kansas School of Medicine-Kansas City \\ Department of Internal Medicine, Division of Hematology and Oncology
}

\begin{abstract}
Background. The purpose of this study was to quantify the prevalence of cancer patients utilizing social support services while undergoing treatment and to identify patient and clinical factors associated with utilization of such services.

Methods. This was a cross-sectional study. Surveys were distributed to three cancer clinics at 11 locations in the greater Kansas City metropolitan area in 2010. Study inclusion criteria included being at least 18 years old and undergoing treatment for cancer at the time of survey completion. Results. A total of 465 oncology patients completed surveys. Two-thirds $(67.5 \%, \mathrm{n}=314)$ were undergoing treatment for cancer and were included in the final analysis. More than half (63.7\%, $n=198)$ were female, and the average age was $58.9 \pm 13.3$ years. More than one-third $(37.4 \%, n$ = 117) reported using cancer-related social support services. Additionally, 22\% $(\mathrm{n}=69)$ reported not using support services but were interested in learning more about those services. Patients had increased odds of having used support services if they were female $(\mathrm{OR}=2.67 ; 95 \% \mathrm{CI}=1.47$, 4.82 ), were younger adults, or had stage I-III (OR $=2.67$; 95\% CI 1.32, 5.26) or stage IV cancer $(\mathrm{OR}=2.3 ; 95 \%$ CI 1.14, 4.75) compared to those who did not know their cancer stage.

Conclusions. More than one-third of patients reported using social support services. A substantial portion of participants reported not using support services but were interested in learning more about those services. Increasing social support service utilization might be especially important to explore for men, those who do not know their cancer stage, and older adults.
\end{abstract}

KS J Med 2014; 7(4):139-148.

\section{Introduction}

In the United States, approximately 1.6 million new cancers are diagnosed annually, and cancer remains the second mostcommon cause of death after heart disease. ${ }^{1}$ Cancer and its treatment can lead to physical disability, psychological distress, and increased healthcare needs. ${ }^{2}$ People diagnosed with cancer experience many physical, family, emotional, practical, and spiritual needs during the acute treatment and chronic management of their cancer. ${ }^{3}$ Services and programs have been developed to address these needs. Patients consider these services as part of high quality cancer care and expect cancer therapy providers to address their supportive care needs. ${ }^{4}$

Social support services are an important factor contributing to decreased distress and improved psychosocial adjustment among cancer patients at all stages of the disease trajectory. ${ }^{5}$ Social support services can be defined as services or programs that help a person with cancer and their families cope with cancer, from pre-diagnosis through 
treatment and cure, or death and bereavement. ${ }^{6}$ Bey proposed to incorporate supportive care as part of the continuous care for cancer patients. ${ }^{7}$ These services can be provided through social workers or nonprofit agencies.

The prevalence of utilizing supportive services varied greatly from one study to another. ${ }^{4}$ One study estimated that $8.2 \%$ of active cancer patients utilized one or more support services offered by a social worker during the previous 12 months. ${ }^{8}$ Another study reported that $15 \%$ to $25 \%$ of cancer patients used cancer-related social support services throughout the trajectory of their disease. ${ }^{9}$ Due to the great variability of supportive services utilization and diversity of methods used in different studies, limited research has explored factors associated with under-utilization of such social support services. ${ }^{4}$ Two studies suggested that younger cancer patients (compared to older) may be more inclined to utilize social support services. ${ }^{8,10}$ However, it is unclear whether other factors (e.g., cancer stage) may be associated with utilization of cancersupport services. Identifying these factors may help clinicians and social workers target vulnerable populations who could benefit from cancer-related social supportive services.

The purpose of this study was to determine the prevalence of social support service usage among adult cancer patients who were undergoing cancer therapy. Additionally, this study sought to identify significant factors associated with support services utilization.

\section{Methods}

Participants. This was a cross-sectional study of cancer patients who attended one of three different oncology outpatient clinics at 11 locations in the Kansas City metropolitan area in 2010. The three clinics included Kansas City Cancer Center with seven locations, the University of Kansas Cancer Center with three locations, and the University of Kansas Radiation Oncology Clinic with one location. The inclusion criteria were: being at least 18 years old, undergoing treatment for cancer (chemotherapy, radiation therapy, or both) at the time of survey completion, able to speak English, and able to give informed consent.

Instrument. The primary method of data collection was a two-page survey with 13 multiple-choice questions. Survey questions included patients' demographic information (age, gender), cancer stage (stage I-III, stage IV, and unknown stage), and whether they were undergoing treatment for cancer currently and if so, the type of treatment (chemotherapy, radiation, or both). The survey included an item regarding whether the respondent was using one or more social support services. If patients were not using a service, they were asked to discontinue the survey. For respondents who were utilizing one or more support services, additional multiple-choice questions with open-ended, write-in options included: the specific services utilized (e.g., counseling, support groups, transportation, wellness programs, financial assistance), specific nonprofit agencies that provide the services, service used most often, reasons for using support services (e.g., personal preference, availability, saves money), source of information about the service (e.g., doctor, nurse, social worker), whether the service improved coping ability, the importance of services (rated on 1-10 scale), type of support provided (e.g., emotional, spiritual, physical), whether the respondent discussed support services with the doctor and/or nurse, and their opinions of whether every cancer clinic should offer information about services to patients.

The primary outcome measure in this study was utilization of one or more social support services. There were four possible 
responses to the primary outcome: (1) "Yes, I use one or more social support services," (2) "No, I do not use support services but I WOULD be interested in learning more about them," (3) "No, I do not use support services, and I would NOT be interested in learning more about them," and (4) "Unsure”. Response options (2), (3), and (4) were categorized as "does not use a support service," and (1) was coded as "uses at least one support service." Additionally, if the respondent checked any of the 19 listed support services or responded to an openended question regarding their use of the support services, the respondent was coded as "uses at least one support service".

Variables associated with support service utilization included gender (male vs female), age (continuous variable), cancer stage (stage I-III, stage IV, and did not know cancer stage $)^{11}$, time since original diagnosis ( $<3$ months, 3 months to 1 year, and $\geq 1$ year), type of treatment received (chemotherapy, radiation, or both), and location of treatment. The location of treatment was used to determine if difference in service utilization occurred by site, but it also served as a proxy for the presence of a full-time social worker. Two clinic locations employed full-time social workers, whereas the third relied on nurses to provide information.

Procedures. Surveys were distributed to three different cancer clinics, both academic and non-academic, at 11 locations in the greater Kansas City metropolitan area. Surveys were completed voluntarily. The specific distribution method at each clinic varied according to the policies of the respective clinical location. Generally, surveys were placed on waiting room tables, completed by patients while waiting in the exam room, or solicited for completion by a single investigator who spent about six days in the waiting rooms at high-volume cancer clinics. Completion of the survey was considered informed consent for the study. This study was approved by the Human Subjects Committee at the University of Kansas Medical Center and the Institutional Review Boards at each clinic.

Statistical analysis. All analyses were conducted using SAS software for Windows (version 9.3, Cary, North Carolina). Descriptive statistics were presented as frequencies and proportions for categorical variables, and means and standard deviations were presented for continuous variables. A Chi-square analysis was conducted to identify the association of demographic variables with the reported support-service utilization. Analysis of variance (ANOVA) was conducted to identify whether there was age differences among three different support service utilization groups. Logistic regression analysis was conducted to identify factors associated with support service utilization (dichotomous variable, utilized one or more support services vs did not utilize support service). The possible predictors included: gender, age, and cancer stage, time since the original diagnosis, type of treatment, and location of receiving treatment. Odds ratios (ORs) with 95\% confidence intervals (CIs) were reported. To obtain a meaningful interpretation of the odds ratio for age, 5year increments were used.

The initial selection of potentially significant predictors was conducted using simple logistic regression with the sole factor included in the model. The significance level was set as 0.10 . With the potentially significant predictors identified, a multiple logistic regression analysis was conducted to identify the final significant predictors. The interaction terms were included in the initial logistic regression model. If the interaction terms were not significant, they were removed from the logistic regression model and only the main effect was included in the final model. The 
Hosmer-Lemeshow goodness-of-fit test assessed how well the logistic regression model fit the data. The significance level for the multiple logistic regression analysis was set as 0.05 . All tests were two-sided.

\section{Results}

Among the 465 respondents who completed the survey, 314 were undergoing cancer treatment and included in the final analysis. Table 1 presents demographic information about these participants. The average age of respondents was $58.9 \pm 13.3$ years. Almost two-thirds (63.7\%, $\mathrm{n}=198$ ) of the respondents were female, $58.2 \%$ reported being 41 to 65 years, and $68.4 \%$ reported having stage I-III or IV cancer ( $\mathrm{n}=$ 210). Additionally, 44.5\% $(\mathrm{n}=138)$ of respondents reported having cancer for more than one year since the original diagnosis, and most patients (72.2\%) were undergoing chemotherapy. A slim majority of patients (51.8\%) were treated at Kansas City Cancer Center. More than one-third of respondents (37.4\%, $n=117$ ) reported using one or more social support services while undergoing treatment for cancer, yet a slightly larger proportion $(40.3 \%, n=126)$ reported not using a support service and reported they were not interested in learning more about the services.

Chi-square analysis was conducted to determine the association between support service utilization status and potential associated factors. Since only one participant reported "unsure" about social support utilization, that category was excluded from the Chi-square analysis. Table 2 presents the results of the Chisquare analysis. A significantly larger proportion of women than men reported using one or more support services (46.2\% vs $22.3 \%, p<0.01)$. Younger age was associated with a higher proportion of utilizing one or more support service $(71.7 \%$ among those aged 18 to 40 years; $40.2 \%$ among those aged 41 to 65 years; $26.1 \%$ among those aged 66 to 80 years; and 7.1\% among those aged 81 years or older, $\mathrm{p}<$ 0.01). Compared to patients who did not know their cancer stage (18.9\%), 48.3\% of stage I-III and $41.3 \%$ of stage IV patients reported using one or more social support service $(p<0.01)$. There was no difference in the utilization of social support services based on patients' duration since their original diagnosis $(p=0.96)$, the type of treatment utilized ( $p=0.64)$, or location of the clinic $(p=0.79)$.

The results of the logistic regression analysis are presented in Table 3. The three factors identified in the Chi-square analysis, gender, age, and cancer stage, remained significantly associated with support service utilization. No interaction term was significant $(\mathrm{p}=0.34$ for age and gender interaction, $p=0.38$ for age and cancer stage interaction, $\mathrm{p}=0.80$ for gender and cancer stage interaction). Women had increased odds (OR $=2.67$; 95\% CI 1.47, 4.82, $\mathrm{p}<0.01$ ) of utilizing support services compared to men. For every five-year increase in age, there was a 13\% decreased odds of support service utilization ( $p<$ 0.01). Finally, compared to respondents who did not know their cancer disease stage, those with stage I-III (OR $=2.67 ; 95 \% \mathrm{CI}$ $1.32,5.26)$ or stage IV (OR $=2.30$; $95 \%$ CI $1.14,4.75)$ had increased odds of utilizing support services ( $p=0.03$ for the overall cancer stage effect). The Hosmer-Lemeshow goodness-of-fit test yielded a value of 11.51 $(p=0.17)$, which indicated a good fit of the logistic model for the data.

\section{Discussion}

In this study, more than one-third (37.4\%) of cancer patients reported using one or more social service. Women were more likely to utilize social support services compared to men. Younger patients were more likely to utilize support services while 
Table 1. Respondents’ demographics ( $\mathrm{N}=314)$.

\begin{tabular}{|c|c|c|}
\hline Gender & Frequency & Percent \\
\hline Male & 113 & $36.3 \%$ \\
\hline Female & 198 & $63.7 \%$ \\
\hline \multicolumn{3}{|l|}{ Age } \\
\hline 18-40 Years & 28 & $9.0 \%$ \\
\hline 41-65 Years & 181 & $58.2 \%$ \\
\hline 66-80 Years & 88 & $28.3 \%$ \\
\hline$>81$ Years & 14 & $4.5 \%$ \\
\hline \multicolumn{3}{|l|}{ Stage of Cancer } \\
\hline Stages I-III & 118 & $38.4 \%$ \\
\hline Stages IV & 92 & $29.7 \%$ \\
\hline Did not know & 97 & $31.6 \%$ \\
\hline \multicolumn{3}{|l|}{ Time Since Original Diagnosis } \\
\hline Less than 3 months & 47 & $15.2 \%$ \\
\hline 3 months to 1 year & 125 & $40.3 \%$ \\
\hline More than 1 year & 138 & $44.5 \%$ \\
\hline \multicolumn{3}{|l|}{ Type of Treatment } \\
\hline Chemotherapy & 215 & $72.2 \%$ \\
\hline Radiation & 34 & $11.4 \%$ \\
\hline Chemotherapy and Radiation & 49 & $16.4 \%$ \\
\hline \multicolumn{3}{|l|}{ Location } \\
\hline Kansas City Cancer Center & 162 & $51.8 \%$ \\
\hline University of Kansas Cancer Center & 101 & $32.3 \%$ \\
\hline $\begin{array}{r}\text { University of Kansas Radiation } \\
\text { Oncology Clinic }\end{array}$ & 50 & $16.0 \%$ \\
\hline \multicolumn{3}{|l|}{ Social Support Service } \\
\hline Used one or more social support service & 117 & $37.4 \%$ \\
\hline $\begin{array}{r}\text { Did not use a support service, and was } \\
\text { not interested in learning more }\end{array}$ & 126 & $40.3 \%$ \\
\hline $\begin{array}{r}\text { Did not use a support service, but was } \\
\text { interested in learning more }\end{array}$ & 69 & $22.0 \%$ \\
\hline Unsure & 1 & $0.3 \%$ \\
\hline
\end{tabular}


Table 2. Comparison of support service utilization among potential predictors.

\begin{tabular}{|c|c|c|c|c|}
\hline & $\begin{array}{l}\text { Did not use a } \\
\text { support } \\
\text { service, and } \\
\text { was not } \\
\text { interested in } \\
\text { learning more }\end{array}$ & $\begin{array}{l}\text { Did not use a } \\
\text { support } \\
\text { service, but } \\
\text { was interested } \\
\text { in learning } \\
\text { more }\end{array}$ & $\begin{array}{l}\text { Used one } \\
\text { or more } \\
\text { social } \\
\text { support } \\
\text { service }\end{array}$ & $\begin{array}{c}\mathrm{p}^{-} \\
\text {value }\end{array}$ \\
\hline Gender (n; \%) & & & & $<0.01$ \\
\hline Male & $59(52.7 \%)$ & $28(25 \%)$ & $25(22.3 \%)$ & \\
\hline Female & $66(33.5 \%)$ & $40(20.3 \%)$ & $91(46.2 \%)$ & \\
\hline Age & & & & $<0.01$ \\
\hline 18-40 Years & $5(17.9 \%)$ & $3(10.7 \%)$ & $20(71.4 \%)$ & \\
\hline 41-65 Years & $64(35.8 \%)$ & $43(24 \%)$ & $72(40.2 \%)$ & \\
\hline 66-80 Years & $50(56.8 \%)$ & $15(17 \%)$ & $23(26.1 \%)$ & \\
\hline$\geq 81$ Years & $6(42.9 \%)$ & $7(50 \%)$ & $1(7.1 \%)$ & \\
\hline Age in Years (Mean \pm SD) & $54.4 \pm 13$ & $59.7 \pm 14$ & $62.5 \pm 12.3$ & $<0.01$ \\
\hline Cancer Stage ( n; \%) & & & & $<0.01$ \\
\hline Stages I-III & $37(31.4 \%)$ & $24(20.3 \%)$ & $57(48.3 \%)$ & \\
\hline Stage IV & $33(35.9 \%)$ & $21(22.8 \%)$ & $38(41.3 \%)$ & \\
\hline Did not know stage & $54(56.8 \%)$ & $23(24.2 \%)$ & $18(18.9 \%)$ & \\
\hline Time Since Original Diagnosis (n;\%) & & & & 0.96 \\
\hline Less than 3 months & $20(42.6 \%)$ & $11(23.4 \%)$ & $16(34 \%)$ & \\
\hline 3 months to 1 year & $47(38.2 \%)$ & $28(22.8 \%)$ & $48(39 \%)$ & \\
\hline More than 1 year & $58(42 \%)$ & $29(21 \%)$ & $51(37 \%)$ & \\
\hline Type of Treatment (n; \%) & & & & 0.64 \\
\hline Chemotherapy & $87(40.7 \%)$ & $46(21.5 \%)$ & $81(37.9 \%)$ & \\
\hline Chemotherapy and radiation & $16(33.3 \%)$ & $9(18.8 \%)$ & $23(47.9 \%)$ & \\
\hline Radiation & $14(41.2 \%)$ & $9(26.5 \%)$ & $11(32.4 \%)$ & \\
\hline Location (n; \%) & & & & 0.79 \\
\hline Kansas City Cancer Center & $70(43.5 \%)$ & $32(19.9 \%)$ & $59(36.6 \%)$ & \\
\hline University of Kansas Cancer Center & $38(37.6 \%)$ & $24(23.8 \%)$ & $39(38.6 \%)$ & \\
\hline $\begin{array}{l}\text { University of Kansas Radiation } \\
\text { Oncology Clinic }\end{array}$ & $18(36.7 \%)$ & $13(26.5 \%)$ & $18(36.7 \%)$ & \\
\hline
\end{tabular}

Table 3. Odds Ratio Estimates with 95\% Confidence Interval for Service Utilization

\begin{tabular}{|c|c|c|}
\hline Effect & Unadjusted OR & Adjusted OR \\
\hline \multicolumn{3}{|l|}{ Gender } \\
\hline Male & Reference & Reference \\
\hline Female & 2.98 (95\% CI 1.77, 5.05) & 2.67 (95\% CI 1.49, 4.91) \\
\hline Age (based on 5-year increments) & 0.81 (95\% CI 0.74, 0.89) & 0.87 (95\% CI 0.78, 0.96) \\
\hline \multicolumn{3}{|l|}{ Cancer Stage } \\
\hline Did not know stage & Reference & Reference \\
\hline Stage I-III & 4.00 (95\% CI 2.13, 7.49) & 2.67 (95\% CI 1.32, 5.26) \\
\hline Stage IV & 3.01 (95\% CI 1.56, 5.82) & 2.30 (95\% CI 1.14, 4.75) \\
\hline
\end{tabular}


undergoing cancer treatment than older patients. Those who knew their cancer stage were more likely to utilize the social support service compared to those who did not know their cancer stage. Duration since the patient's original diagnosis, treatment type, and treatment site were not associated with utilization of cancer-related support services.

The reported $37.4 \%$ prevalence of social support utilization was higher than what has been reported in the literature (15-25\%). ${ }^{9}$ This difference may be attributable to multiple factors, including different definitions of social support services, ${ }^{6,7}$ differences in the types of social support services (e.g., psychological, physical, spiritual) that were eligible to be included in the study, ${ }^{4}$ and availability of social support services for cancer patients in different regions and different types of cancer. $^{4}$ Additionally, our study suggested there was a small but significant proportion (22\%) of cancer patients who reported interest in learning more about available social support services, suggesting that study participants were unaware of available services. As such, cancer clinics might need to re-evaluate their method of informing patients about available services.

This study identified factors associated with social support utilization during cancer treatment including younger, female, and knowing one's cancer stage. Few studies had evaluated the prevalence of support services utilization during treatment.,3,12,13 Our results were similar to these studies as patients tended to have higher needs if they were younger, female, or had cancer that was not in remission.

Cancer stage was a significant factor for utilization of support services. Patients with advanced pathological cancer stages have higher patient care and support needs in the acute treatment phase than patients with less advanced cancer. ${ }^{14}$ In this study, stage IV cancer patients had lower odds of social support services utilization compared to stage I-III cancer patients. This unexpected finding may be explained by the selfreporting mechanism of supportive service utilization. Additional research is warranted to explore why a significant subset (32\%) of cancer patients did not know their cancer stage, even when given multiple choice options. More importantly, lack of knowledge regarding disease stage may be a proxy indicator of lower health literacy and/or lack of self-efficacy following a cancer diagnosis. These patients may have a higher degree of fatalism, and fail to see any benefit of social support utilization. Healthcare providers should make sure all patients, but especially those with possible lower selfefficacy, are aware of cancer social support services and the specific types of support provided.

The current study found that time since cancer diagnosis was not a significant predictor for support service utilization. However, previous studies suggested that patients are more likely to utilize services during the initial time period after diagnosis due to emotional distress, psychological distress, and level of unmet needs. ${ }^{2,10,14}$ Patients might need to be assessed regularly throughout treatment and thereafter to evaluate unmet needs that could be alleviated through support services. Research that would track the dynamic utilization of support services by cancer patients over time could elucidate nuances regarding the timing of service needs and utilization.

In contrast to similar studies, treatment type (chemotherapy, radiation, or both) was not associated with utilization. Previous research suggested that compared to no treatment, those undergoing chemotherapy are more likely to have unmet needs. ${ }^{15,16}$ In 
our study, patients who received neoadjuvant/adjuvant treatment were not compared to those who received surgery or did not undergo any form of treatment. In addition, some patients undergoing chemotherapy may report unmet needs even if they utilized social support services. Thus, our outcome may not be completely comparable with the presence of unmet needs.

Finally, there was no difference in service utilization by treatment location, suggesting that the treatment sites were similar in this regard. The presence of a fulltime social worker did not increase the social support utilization by cancer patients. This lack of difference may be attributed to the various locations' relatively equal time allocation by the nurse/social worker dedicated to disseminating support service information. No previous studies had investigated the utilization of support services by social worker presence in the clinic. Future research could identify the barriers to using social support services even when the social workers are present in the clinic.

Limitations. The study data were selfreported and subject to reporting bias. The methods of survey distribution were inconsistent across clinic location and dependent upon each clinic's policies. The survey did not include self-reported demographic questions. For instance, the instrument did not include race, ethnicity, educational level, income, health insurance status, type of cancer, or current length of the treatment. Additionally, the instrument did not assess patients' treatment frequency, overall duration, or cancer type. These variables would be important to identify associations with utilization of cancerrelated support services. Finally, the instrument did not collect clinical outcome data such as survival, quality of life improvement, and decreased psychological morbidity. Therefore, the results of this study should be considered hypothesis generating. Research is needed to evaluate the medical benefit of social support services and to elucidate which characteristics of patients who do not utilize social support services but may have unmet needs. Finally, the definition of "social support services" was broad. Different types and stages of cancer patients have expressed different needs. ${ }^{4}$ Even the same type of cancer patients will express different needs. ${ }^{17,18}$ Cawley et al. ${ }^{18}$ listed information seeking as the unmet need for breast cancer patients, whereas Girgis et al. ${ }^{17}$ listed information seeking, disease specific needs, and psychological and psychosocial needs. As expected, the needs for mixed types of cancer patients will vary greatly by cancer type and stage.

\section{Conclusions}

More than one-third of cancer patients reported using social support services while undergoing cancer treatment. Women and younger patients reported a higher prevalence of cancer-related support service utilization, as did those with stage I-IV cancer (compared to those who did not know their cancer stage). More than half of cancer patients utilized or were interested in learning more about social support services. Therefore, cancer clinics must be prepared to provide information about the availability and the specific types of services provided to cancer patients as an integral component of quality cancer care. Future research is needed to understand the potential unmet needs of all sub-populations, especially men, older adults, and patients who do not know their cancer stage to increase the quality of life of patients following a diagnosis of cancer. 


\section{References}

1 American Cancer Society. Cancer facts and figures. New York: American Cancer Society, 2012.

${ }^{2}$ Puts MT, Papoutsis A, Springall E, Tourangeau AE. A systematic review of unmet needs of newly diagnosed older cancer patients undergoing active cancer treatment. Support Care Cancer 2012; 20(7):1377-1394. PMID: 22476399.

${ }^{3}$ Sanson-Fisher R, Girgis A, Boyes A, Bonevski B, Burton L, Cook P. The unmet supportive care needs of patients with cancer. Supportive Care Review Group. Cancer 2000; 88(1):226-237. PMID: 10618627.

${ }^{4}$ Harrison JD, Young JM, Price MA, Butow $\mathrm{PN}$, Solomon MJ. What are the unmet supportive care needs of people with cancer? A systematic review. Support Care Cancer 2009; 17(8):1117-1128. PMID: 19319577.

${ }^{5}$ Kornblith AB, Herndon JE $2^{\text {nd }}$, Zuckerman E, et al. Social support as a buffer to the psychological impact of stressful life events in women with breast cancer. Cancer 2001; 91(2):443-454. PMID: 11180093.

${ }^{6}$ Gysels M, Higginson IJ, Rajasekaran M, Davies E, Harding R. Improving supportive and palliative care for adults with cancer. Research Evidence Manual. London, England: National Institute of Clinical Excellence, 2004.

7 Bey P. Future trends in oncology. Support Care Cancer 2000; 8(2):98-101. PMID: 10739355.

8 Gadalla TM. Cancer patients' use of social work services in Canada: Prevalence, profile, and predictors of use. Health Soc Work 2007; 32(3):189-196. PMID: 17896675.

${ }^{9}$ Cwikel JG, Behar LC. Organizing social work services with adult cancer patients: Integrating empirical research. Soc Work
Health Care 1999; 28(3):55-76. PMID: 10457981.

${ }^{10}$ Andersen BL. Psychological interventions for cancer patients to enhance the quality of life. J Consult Clin Psychol 1992; 60(4):552-568. PMID: 1506503.

${ }^{11}$ Greene FL, Page DL, Fleming ID, et al. AJCC Cancer Staging Handbook. From the AJCC Cancer Staging Manual. Sixth Edition. New York: Springer Verlag, 2002.

${ }^{12}$ McIllmurray MB, Thomas C, Francis B, Morris S, Soothill K, Al-Hamad A. The psychosocial needs of cancer patients: Findings from an observational study. Eur J Cancer Care (Engl) 2001; 10(4):261-269. PMID: 11806677.

${ }^{13}$ Matsuyama RK, Kuhn LA, Molisani A, Wilson-Genderson MC. Cancer patients' information needs the first nine months after diagnosis. Patient Educ Couns 2013; 90(1):96-102. PMID: 23058682.

${ }^{14}$ Chen SC, Liao CT, Lin CC, Chang JT, Lai YH. Distress and care needs in newly diagnosed oral cavity cancer patients receiving surgery. Oral Oncol 2009; 45(9):815-820. PMID: 19250858.

${ }^{15}$ Veloso AG, Sperling C, Holm LV, et al. Unmet needs in cancer rehabilitation during the early cancer trajectory-A nationwide patient survey. Acta Oncol 2013; 52(2):372-381. PMID: 23320774.

${ }^{16}$ Boyes AW, Girgis A, D’Este C, Zucca AC. Prevalence and correlates of cancer survivors' supportive care needs 6 months after diagnosis: A population-based crosssectional study. BMC Cancer 2012; 12:150. PMID: 22510387.

${ }^{17}$ Girgis A, Boyes A, Sanson-Fisher RW, Burrows S. Perceived needs of women diagnosed with breast cancer: Rural versus urban location. Aust N Z J Public Health 2000;2 4(2):166-173. PMID: 10790936. 
${ }^{18}$ Cawley M, Kostic J, Cappello C. Keywords: cancer, social support, Informational and psychosocial needs of women choosing conservative surgery/ primary radiation for early stage breast cancer. Cancer Nurs 1990; 13(2):90-94. PMID: 2331696.

psychosocial factors, cancer treatment protocol, needs assessment 